\title{
Early Osmotherapy in Severe Traumatic Brain Injury : An International Multicenter Study
}

\section{TBI Collaborative}

2020-01

TBI Collaborative , Anstey , J R , Taccone , F S , Udy , A A \& Skrifvars , M B 2020 , ' Early Osmotherapy in Severe Traumatic Brain Injury : An International Multicenter Study ' , Journal of Neurotrauma, vol. 37 , no. 1 , pp. 178-184 . https://doi.org/10.1089/neu.2019.6399

http://hdl.handle.net/10138/319698

https://doi.org/10.1089/neu.2019.6399

unspecified

publishedVersion

Downloaded from Helda, University of Helsinki institutional repository.

This is an electronic reprint of the original article.

This reprint may differ from the original in pagination and typographic detail.

Please cite the original version. 


\title{
Early Osmotherapy in Severe Traumatic Brain Injury: An International Multicenter Study
}

\author{
James R. Anstey,, Fabio S. Taccone, ${ }^{2}$ Andrew A. Udy, ${ }^{3,16}$ Giuseppe Citerio, ${ }^{4}$ Jacques Duranteau, ${ }^{5}$ \\ Carole Ichai, ${ }^{6}$ Rafael Badenes, John R. Prowle, ${ }^{8}$ Ari Ercole, ${ }^{9}$ Mauro Oddo, ${ }^{10}$ Antoine G. Schneider, \\ Mathieu van der Jagt, ${ }^{11}$ Stefan Wolf, ${ }^{12}$ Raimund Helbok, ${ }^{13}$ David W. Nelson, ${ }^{14}$ Marius B. Skrifvars, ${ }^{15}$ \\ Anatole Harrois, ${ }^{1,5}$ Jeffrey Presneill, ${ }^{1}$ D. Jamie Cooper, ${ }^{3,16}$ Michael Bailey, ${ }^{16,17}$ and Rinaldo Bellomo ${ }^{1,16,18}$ \\ on behalf of The TBI Collaborative
}

\begin{abstract}
The optimal osmotic agent to treat intracranial hypertension in patients with severe traumatic brain injury (TBI) remains uncertain. We aimed to test whether the choice of mannitol or hypertonic saline (HTS) as early (first $96 \mathrm{~h}$ ) osmotherapy in these patients might be associated with a difference in mortality. We retrospectively analyzed data from 2015 from 14 tertiary intensive care units (ICUs) in Australia, UK, and Europe treating severe TBI patients with intracranial pressure (ICP) monitoring and compared mortality in those who received mannitol only versus HTS only. We performed multivariable analysis adjusting for site and illness severity (Injury Severity Score, extended IMPACT score, and mean ICP over the first $96 \mathrm{~h}$ ) using Cox proportional hazards regression. We collected data on 262 patients and compared patients who received early osmotherapy with mannitol alone $(n=46)$ with those who received HTS alone $(n=46)$. Mannitol patients were older (median age, 49.2 (19.2) vs. 40.5 (16.8) years; $p=0.02$ ), with higher Injury Severity Scores (42 (15.9) vs. 32.1 [11.3]; $p=0.001)$, and IMPACT-TBI predicted 6-month mortality (34.5\% [23-46] vs. 25\% [13-38]; $p=0.02)$, but had similar APACHE-II scores, and mean and maximum ICPs over the first $96 \mathrm{~h}$. The unadjusted hazard ratio for inhospital mortality in patients receiving only mannitol was 3.35 (95\% confidence interval $[\mathrm{CI}], 1.60-7.03 ; p=0.001)$. After adjustment for key mortality predictors, the hazard ratio for in-hospital mortality in patients receiving only mannitol was 2.64 (95\% CI, 0.96-7.30; $p=0.06)$. The choice of early osmotherapy in severe TBI patients may affect survival, or simply reflect clinician beliefs about their different roles, and warrants controlled investigation.
\end{abstract}

Keywords: hypertonic saline; mannitol; mortality; osmotherapy; traumatic brain injury

${ }^{1}$ Intensive Care Unit, Royal Melbourne Hospital, Parkville, Victoria, Australia.

${ }^{2}$ Department of Intensive Care, Erasme Hospital, Université Libre de Bruxelles, Brussels, Belgium.

${ }^{3}$ Intensive Care Unit, The Alfred Hospital, Melbourne, Victoria, Australia.

${ }^{4}$ School of Medicine and Surgery, University Milano Bicocca-Neurointensive Care, San Gerardo Hospital, ASST-Monza, Monza, Italy.

${ }^{5}$ Department of Anesthesia and Surgical Intensive Care, CHU de Bicetre, Le Kremlin Bicêtre, Le Kremlin-Bicêtre, France.

${ }^{6}$ Université Côte d'Azur, Centre hospitalier Universitaire de Nice, Service de Réanimation polyvalente, Hôpital Pasteur 2, CHU de Nice, Nice, France.

${ }^{7}$ Department of Anesthesiology and Surgical-Trauma Intensive Care, Hospital Clinic Universitari de Valencia, University of Valencia, Valencia, Spain.

${ }^{8}$ Adult Critical Care Unit, The Royal London Hospital, Barts Health NHS Trust, Royal London Hospital, Whitechapel Road, London, United Kingdom.

${ }^{9}$ Neurosciences and Trauma Critical Care Unit, Cambridge University Hospitals NHS Foundation Trust, Cambridge, United Kingdom.

${ }^{10}$ Department of Medical-Surgical Intensive Care Medicine, Faculty of Biology and Medicine, Centre Hospitalier Universitaire, Vaudois (CHUV), University of Lausanne, Lausanne, Switzerland.

${ }^{11}$ Department of Intensive Care, Erasmus MC-University Medical Centre, Erasmus MC-University Medical Center, Rotterdam, The Netherlands.

${ }^{12}$ Department of Neurosurgery, Charité Universitätsmedizin Neuro Intensive Care Unit 102i, Campus Charité Mitte (CCM), Berlin, Germany.

${ }^{13}$ Neurological Intensive Care Unit, Department of Neurology, Medical University of Innsbruck, Department of Neurology, Neurocritical Care Unit, Innsbruck, Austria.

${ }^{14}$ Section for Perioperative Medicine and Intensive Care, Department of Physiology and Pharmacology, Karolinska Institute, Stockholm, Sweden.

${ }^{15}$ Division of Intensive Care, Department of Anaesthesiology, Intensive Care and Pain Medicine and Department of Emergency Medicine and Services, Helsinki University Hospital and University of Helsinki, Helsinki, Finland.

${ }^{16}$ Australian and New Zealand Intensive Care Research Centre, School of Public Health and Preventative Medicine, Monash University, ANZIC-RC, Department of Epidemiology and Preventive Medicine, Monash University, Melbourne, VIC, Australia.

${ }^{17}$ Department of Medicine and Radiology, University of Melbourne, Parkville, Victoria, Australia.

${ }^{18}$ Department of Intensive Care, Austin Health, Melbourne, Victoria, Australia. 


\section{Introduction}

I NTRACRANIAL HYPERTENSION (ICH) can be life-threatening after severe traumatic brain injury (TBI), yet the optimal therapeutic response remains controversial. In particular, the role of hypothermia and decompressive craniectomy has been challenged by recent randomized controlled trials. ${ }^{1-3}$ In contrast, osmotherapy retains an important position in the management of $\mathrm{ICH}^{4}$ Two agents, mannitol and hypertonic saline (HTS), are routinely used in clinical practice to treat this condition. However, consensus guidelines do not provide direction for the selection of a specific agent. ${ }^{4,5}$ Moreover, a recent systematic review has failed to resolve this issue, because of limited comparative studies focusing on clinical outcomes. ${ }^{6}$ Finally, recent practice surveys suggest that these two options are equally commonly used. ${ }^{7,8}$

Given the lack of evidence-supported options for severe ICH, it seems logical to focus research on the optimal choice of osmotherapy. In particular, it is now important to assess the epidemiology of modern osmotherapy use, including the choice of agent, the volume and timing of administration (especially in the early phase of ICH), and any association between choice of agent and mortality.

Accordingly, we performed an international, retrospective, multicenter study of the management of severe TBI, focusing on the epidemiology of osmotherapy and, in particular, the choice, volume, and timing of early osmotherapy. We aimed to test the hypothesis that the choice of HTS or mannitol as osmotherapy in patients with TBI and ICH might be associated with a difference in in-hospital mortality.

\section{Methods}

\section{Study design and data}

We performed a retrospective study involving 14 tertiary ICUs in Australia and Europe, each treating a high volume of TBI patients. Ethics approval for contribution to this international data set was obtained locally by each center.

Two centers were from Australia (both from Melbourne), two were from the UK (London and Cambridge), and the remaining 10 were from continental Europe (Paris and Nice, France; Valencia, Spain; Lausanne, Switzerland; Brussels, Belgium; Monza, Italy; Berlin, Germany; Rotterdam, The Netherlands; Solna, Sweden; and Innsbruck, Austria).

Centers contributed data from severe TBI (Glasgow Coma Scale [GCS] $\leq 8$ post-resuscitation) patients in 2015 with an intracranial pressure (ICP) monitor in situ, up to a maximum of 20 patients per center. Eligible patients were of at least 18 years of age, with either an isolated severe TBI or severe TBI as part of multi-trauma, and had an ICP monitor for at least $96 \mathrm{~h}$. Baseline demographic data (age, indicators of TBI severity [neurological and vital signs prehospital and on hospital arrival], initial computed tomography [CT] scan findings as summarized by the Marshall score, and mean ICPs over the first $96 \mathrm{~h}$ ), and illness severity scores (APACHE-II, Injury Severity Score [ISS]) were collected for each patient. An extended IMPACT score (core + CT + lab) - a prognostic tool of 6-month outcome after moderate and severe $\mathrm{TBI}^{9}$ - was calculated for each patient.

Six-hourly ICPs, as recorded from the extraventricular drain or intraparenchymal catheter, as well as aspects of neurointensive care management, were recorded for all patients. Data were collected for up to 7 days from ICU admission, or until the ICP monitor was removed, whichever came first.

Outcome data included in-hospital mortality, length of ICU and hospital stay, and days alive and free from ICU at 30 days, and days alive and free from hospital at 60 days. Those patients who received osmotherapy with mannitol only or HTS only during the first $96 \mathrm{~h}$ of their ICU stay were compared with regard to mortality. Groups were separated in this way to avoid the confounding effect of exposure to both agents. This time cutoff was chosen because, as previously reported, most episodes of osmotherapy treatment in severe TBI patients take place during this period. ${ }^{10}$

In order to standardize the different volumes and concentrations of HTS and mannitol delivered, we calculated the osmotic load. For example, $100 \mathrm{~mL}$ of $3 \% \mathrm{NaCl}(1027 \mathrm{mOsm} / \mathrm{L})$ has a roughly equivalent osmotic load to $100 \mathrm{~mL}$ of $20 \%$ mannitol $(1100 \mathrm{mOsm} / \mathrm{L})$, both being approximately $100 \mathrm{mOsm}$.

\section{Statistical analysis}

All data were initially assessed for normality. Group comparisons were performed using chi-square tests for equal proportion, Student $t$-tests for normally distributed data, and Wilcoxon ranksum tests otherwise, with results reported as $n(\%)$, mean (standard deviation), or median (interquartile range; IQR) respectively.

The primary outcome (hospital mortality) was analyzed using Cox/proportional hazards regression. To account for known predictors of outcome and potential heterogeneity between sites, multi-variable regression was performed adjusting for ISS, extended IMPACT (core + lab + CT) score, mean ICP over the first $96 \mathrm{~h}$, and center, with the latter treated as a random effect.

To further account for baseline imbalance between mannitol and HTS groups, propensity-adjusted sensitivity analysis was performed. Using multi-variable logistic regression with exclusive mannitol usage as the outcome, a model was derived to create the probability (propensity) that each patient would receive mannitol. This model was developed using both step-wise selection and backward elimination techniques, incorporating only variables that had a $p$ value $<0.2$ for both techniques. Baseline variables considered for model inclusion were age, sex, APACHE-II score, ISS, and neurosurgery for clot evacuation. Each patient's propensity to receive mannitol was then included as a covariate in the multi-variable models in conjunction with treatment, extended IMPACT score, mean ICP over the first $96 \mathrm{~h}$, and center (random effect). Time to event between the two groups was presented using Kaplan-Meier curves with comparison using log-rank tests.

All analysis was performed using SAS software (version 9.4; SAS Institute Inc., Cary, NC), and a two-sided $p$ value of 0.05 was used to indicate statistical significance.

\section{Results \\ Patient selection}

We collected data on 262 consecutive patients. We excluded patients who died within the first $96 \mathrm{~h}$, as well as those who did not receive osmotherapy during this period. Moreover, we excluded patients who were exposed to both mannitol and HTS during the study period. Accordingly, 46 patients received mannitol only, and 46 patients received HTS only during this period (Fig. 1).

The characteristics of the two osmotherapy groups are shown in Table 1. In comparison, patients who received no early osmotherapy whatsoever had similar ISS, APACHE-II scores, and extended IMPACT scores, but lower daily maximum ICPs, than those who received osmotherapy (see Supplementary Table S1)

Patients were predominantly young to middle-aged males in both groups, with a significantly older age in those treated with mannitol. Further, illness severity scores (ISS, APACHE-II scores, and extended IMPACT scores) indicated greater severity in those patients treated with mannitol. Finally, although more patients in the mannitol group had neurosurgery for clot evacuation, there was no difference in the rates of decompressive craniectomy or extraventricular drainage of CSF. 


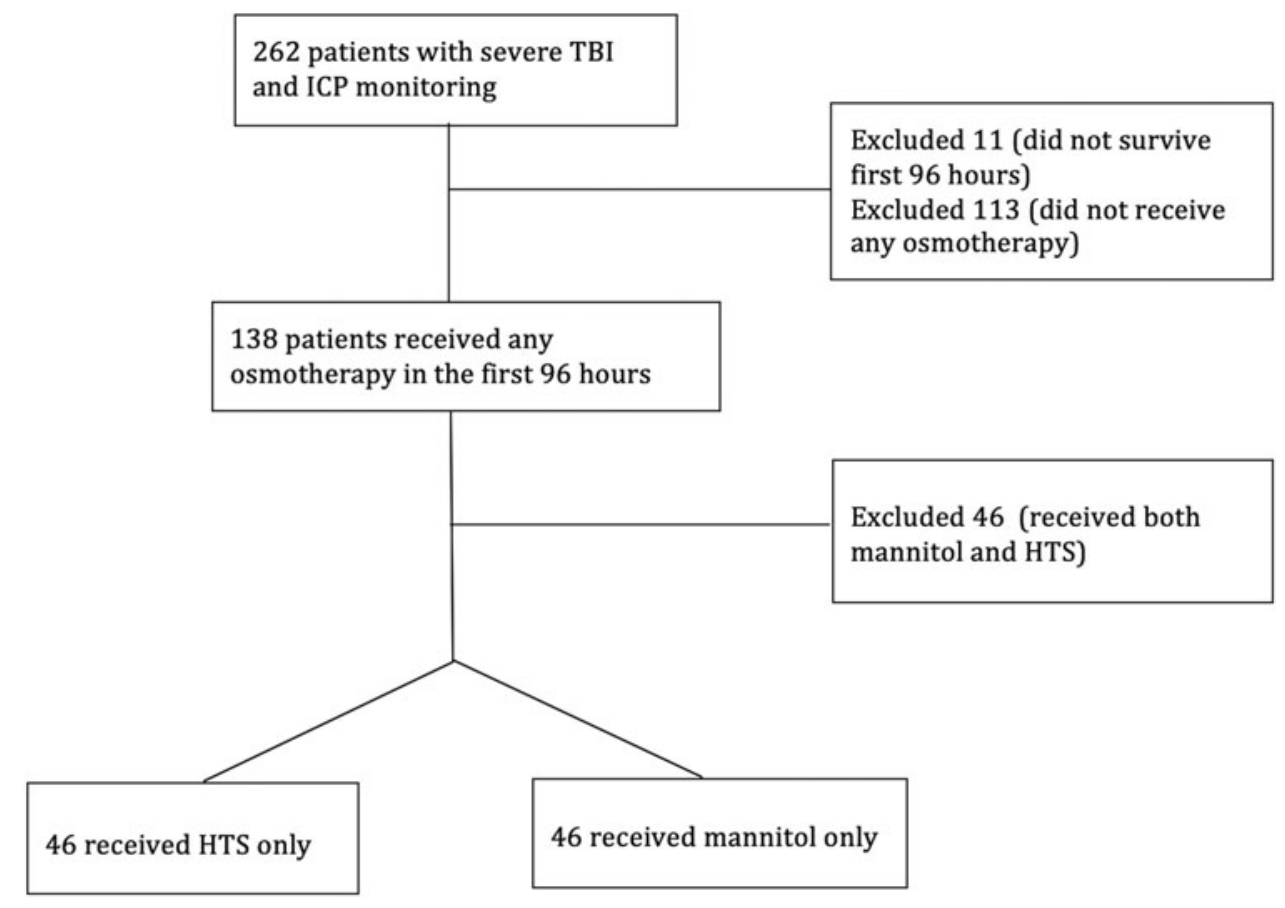

FIG. 1. A flow chart of the identification of patients who received only one type of osmotherapy: hypertonic saline or mannitol, in the first $96 \mathrm{~h}$ of ICU stay. HTS, hypertonic saline; ICP, intracranial pressure; ICU, intensive care unit; TBI, traumatic brain injury.

Hypothermia and barbiturate coma were used in similar amounts in both groups (see Table 1). Moreover, propofol was used in a greater percentage of patients treated with HTS. Importantly, mean and maximum ICPs over the first $96 \mathrm{~h}$ were similar in the two groups (see Fig. 2).

\section{Osmotherapy}

All 14 centers used osmotherapy in certain patients within the study time window. However, two centers used only HTS, two centers used only mannitol, and 10 centers used a combination of both. Each center contributed a median of 3 [IQR, 0-5] patients to the HTS group and 3 [IQR, 0-5] patients to the mannitol group.

HTS concentration varied by center; however, each center used only one HTS concentration. The range of HTS concentrations included 3\% ( $n=3), 5 \% \quad(n=2), 7 \% \quad(n=1), 7.5 \% \quad(n=3), 10 \%$ $(n=2)$, and $23.5 \% \mathrm{NaCl}(n=1)$. Approximately half of the HTS group received osmotherapy on any given day, as described in Table 2, for a median total osmotic load of 629 [322-965] mOsm during the first $96 \mathrm{~h}$.

Twenty percent mannitol was the formulation used in the 11 centers using mannitol, while one center used 15\% mannitol. Mannitol use was greatest on the first day, whereas it was less commonly used for the remainder of the week, with approximately one quarter of patients receiving such therapy on any given day. Patients received a median total osmotic load of 447[238-745] mOsm of mannitol during the first $96 \mathrm{~h}$. Hence, patients in the HTS group received a larger median osmotic load than those in the mannitol group (see Table 2).

\section{Outcomes}

Patients receiving mannitol only were 3 times more likely to die in the hospital than patients receiving HTS only (hazard ratio [95\% confidence interval $\{\mathrm{CI}\}], 3.35$ [1.60-7.03]; $p=0.001)$. However, after accounting for adjustment for center, ISS, extended IMPACT, and mean ICP over the first $96 \mathrm{~h}$, this result no longer retained statistical significance (hazard ratio [95\% CI], 2.64 [0.96-7.30]; $p=0.06$; Table 3).

When the multi-variable analysis of time to death was additionally adjusted for each patient's probability (propensity) to receive mannitol, the increased risk in death for mannitol patients remained non-significant (hazard ratio [95\% CI], 2.52 [0.90-7.06]; $p=0.08$; Supplementary Tables S2 and S3).

Table 4 summarizes unadjusted hospital and ICU mortality, days alive, and free from ICU to 30 days, and days alive and free from hospital to 60 days, censored at hospital discharge, according to osmotherapy. Hospital mortality was more than twice as great in the mannitol-only group, whereas unadjusted ICU mortality was 3 times greater in this group. Consistent with these findings, the number of days alive and free from ICU and hospital was also greater in the HTS group. Unadjusted time to death is further illustrated in Figure 3.

\section{Discussion}

\section{Key findings}

We performed an international observational, multi-center study of patients with severe TBI, ICP monitoring and ICH. We assessed the epidemiology of osmotherapy use, with focus on the choice, volume, and timing of early osmotherapy, and aimed to test the hypothesis that the exclusive use of mannitol or HTS would be associated with a difference in mortality. We found that patients treated with mannitol were more severely injured. Moreover, we found that, in such mannitol-treated patients, on unadjusted comparison, both ICU and hospital mortality were significantly higher. Importantly, we observed that the two agents were used differently in terms of timing, with mannitol being used predominantly on the 
Table 1. Comparison of Baseline Characteristics, Injury Severity, and Treatments of Patients Receiving HTS Only versus Mannitol Only in the First 96 h of ICU Stay

\begin{tabular}{|c|c|c|c|}
\hline & HTS only $(\mathrm{n}=46)$ & Mannitol only $(\mathrm{n}=46)$ & $\mathrm{p}$ value \\
\hline Characteristics Age in years & $40.5(16.8)$ & $49.2(19.2)$ & 0.02 \\
\hline Male sex, \% (n) & $71.7(33)$ & $76.1(35)$ & 0.64 \\
\hline \multicolumn{4}{|l|}{ Severity of injury } \\
\hline GCS (post-resuscitation, pre-intubation) & $5[3-7]$ & $5[3-9]$ & 0.30 \\
\hline Marshall Score & $3[2-5]$ & $4[3-5]$ & 0.02 \\
\hline Injury Severity Score & $32.1(11.3)$ & $42(15.9)$ & 0.001 \\
\hline APACHE II & $20[15-23]$ & $22[17-32]$ & 0.1 \\
\hline Extended IMPACT predicted 6-month $\%$ mortality & $25[13-38]$ & $34.5[23-46]$ & 0.02 \\
\hline Mean ICP over first $96 \mathrm{~h}, \mathrm{~mm} \mathrm{Hg}$ & $13.4(4.3)$ & $13.8(5.2)$ & 0.69 \\
\hline \multicolumn{4}{|l|}{ Maximum ICP by day, $\mathrm{mm} \mathrm{Hg}$} \\
\hline Day 0 & $16.7(9.0)$ & $19.9(14.4)$ & 0.21 \\
\hline Day 1 & $18.0(7.2)$ & $18.5(8.7)$ & 0.78 \\
\hline Day 2 & $17.2(6.3)$ & $17.1(7.6)$ & 0.95 \\
\hline Day 3 & $16.4(5.5)$ & $19.3(12.4)$ & 0.16 \\
\hline Maximum ICP in the first $96 \mathrm{~h}, \mathrm{~mm} \mathrm{Hg}$ & $22.7(7.4)$ & $26.4(15.9)$ & 0.16 \\
\hline \multicolumn{4}{|l|}{ Treatments } \\
\hline Neurosurgery for clot evacuation, $\%(n)$ & $26.1(12)$ & $47.2(17)$ & 0.05 \\
\hline Decompressive craniectomy, $\%(n)$ & $23.9(11)$ & $21.7(10)$ & 0.80 \\
\hline Day of decompressive craniectomy & $1[0-2]$ & $1[0-2]$ & 0.78 \\
\hline Extraventricular CSF drainage, $\%(n)$ & $45.7(21)$ & $43.5(20)$ & 0.83 \\
\hline \multicolumn{4}{|l|}{ Serum $\mathrm{Na}^{+}, \mathrm{mmol} / \mathrm{L}$} \\
\hline Day 0 & $142(4.9)$ & $142(6.3)$ & 1.0 \\
\hline Day 1 & $143(5.2)$ & $142(6.5)$ & 0.42 \\
\hline Day 2 & $144(5.2)$ & $143(6.6)$ & 0.42 \\
\hline Day 3 & $144(5.2)$ & $143(6.6)$ & 0.42 \\
\hline \multicolumn{4}{|l|}{$\mathrm{PaCO}_{2}, \mathrm{~mm} \mathrm{Hg}$} \\
\hline Day 0 & $36.7(6.0)$ & $37.9(6.3)$ & 0.35 \\
\hline Day 1 & $36.9(5.8)$ & $36.9(5.1)$ & 1.0 \\
\hline Day 2 & $37.3(4.7)$ & $37.2(5.2)$ & 0.92 \\
\hline Day 3 & $36.9(4.3)$ & $38.5(5.2)$ & 0.11 \\
\hline Sedation with propofol, $\%(n)^{\mathrm{a}}$ & $75.5(34 / 35)$ & $35.1(13 / 37)$ & 0.0003 \\
\hline Sedation with opioid, $\%(n)^{\mathrm{a}}$ & $97.8(44 / 45)$ & $95.6(44 / 46)$ & 0.56 \\
\hline Sedation with midazolam, $\%(n)^{\mathrm{a}}$ & $64.4(29 / 45)$ & $43.5(20 / 46)$ & 0.05 \\
\hline Barbiturate coma, $\%(n)$ & $13(6)$ & $19.6(9)$ & 0.40 \\
\hline Hypothermia $<35^{\circ} \mathrm{C}$ at any point, $\%(n)$ & $39(18)$ & $30(14)$ & 0.38 \\
\hline
\end{tabular}

Values are presented as mean (standard deviation) or median [interquartile range].

${ }^{a}$ Sedation with propofol/opioid/midazolam refers to any use of these agents in isolation or combination during the first $96 \mathrm{~h}$.

CSF, cerebrospinal fluid; CT, computed tomography; HTS, hypertonic saline; ICP, intracranial pressure; ICU, intensive care unit; IMPACT, International Mission for Prognosis and Analysis of Clinical Trials in Traumatic brain injury; TBI, traumatic brain injury.

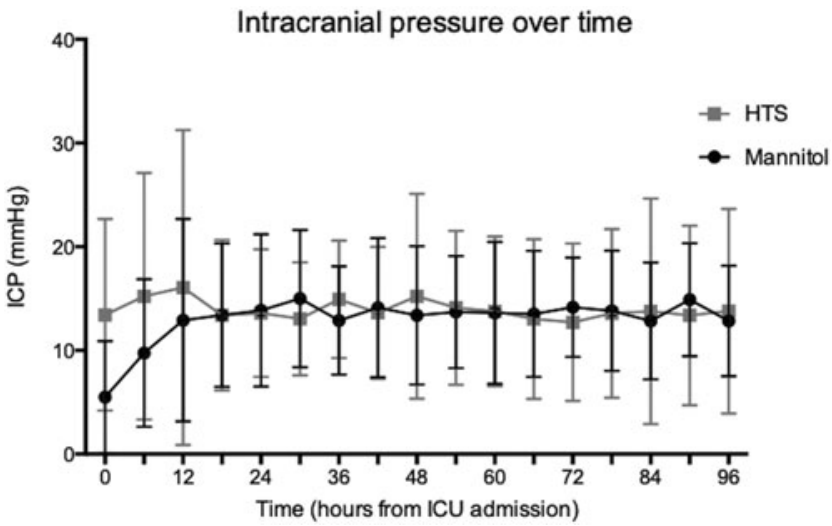

FIG. 2. A comparison of mean intracranial pressure over time between the two groups. Values presented are mean \pm standard deviation. HTS, hypertonic saline; ICP, intracranial pressure; ICU, intensive care unit.
Table 2. Osmotic LoAd Administered BY DAY of TREATMENT

\begin{tabular}{|c|c|c|c|c|}
\hline & \multicolumn{2}{|c|}{$\begin{array}{c}\text { Hypertonic saline } \\
(3.0-23.5 \%)\end{array}$} & \multicolumn{2}{|c|}{ Mannitol (15-20\%) } \\
\hline & $\begin{array}{l}\text { No. treated } \\
\text { that day }\end{array}$ & $\begin{array}{l}\text { Osmotic load } \\
\text { for patients } \\
\text { treated that } \\
\text { day }(\mathrm{mOsm})\end{array}$ & $\begin{array}{l}\text { No. treated } \\
\text { that day }\end{array}$ & $\begin{array}{l}\text { Osmotic load } \\
\text { for patients } \\
\text { treated that } \\
\text { day (mOsm) }\end{array}$ \\
\hline Day 0 & 26 & 325 [189-642] & 35 & $275[22$ \\
\hline Day 1 & 24 & 342 [171-643] & 11 & 281 [220-549] \\
\hline Day 2 & 23 & 257 [171-449] & 13 & 384 [137-549] \\
\hline Day 3 & 15 & 342 [217-513] & 10 & $275[171-412]$ \\
\hline
\end{tabular}

$p$ value $=0.09$ for total osmotic load between groups over first $96 \mathrm{~h}$. $n=$ number of patients receiving osmotherapy on a given day; median and interquartile range of osmotic load are presented.

Osmotic load=total daily mOsm given; $100 \mathrm{~mL}$ of $3 \% \mathrm{NaCl}$ $(1027 \mathrm{mOsm} / \mathrm{L})$ has a roughly equivalent osmotic load to $100 \mathrm{~mL}$ of $20 \%$ mannitol $(1100 \mathrm{mOsm} / \mathrm{L})$. 
Table 3. Unadjusted and Adjusted Hazard Ratios for In-Hospital Mortality

\begin{tabular}{|c|c|c|c|c|}
\hline Variable & $\begin{array}{c}\text { Unadjusted hazard } \\
\text { ratio }[95 \% \mathrm{CI}]\end{array}$ & $\begin{array}{l}\text { Unadjusted } \\
\text { p value }\end{array}$ & $\begin{array}{l}\text { Adjusted hazard } \\
\text { ratio }[95 \% C I]^{\mathrm{a}}\end{array}$ & $\begin{array}{c}\text { Adjusted } \\
\mathrm{p} \text { value }\end{array}$ \\
\hline Mannitol & 3.35 [1.60-7.03] & 0.001 & $2.64[0.96-7.30]$ & 0.06 \\
\hline Injury Severity Score & 1.05 [1.03-1.07] & $<0.0001$ & 1.04 [1.01-1.06] & 0.008 \\
\hline Extended IMPACT Score & $1.03[1.01-1.05]$ & 0.006 & $1.04[1.01-1.06]$ & 0.002 \\
\hline Mean ICP over first $96 \mathrm{~h}$ & 1.09 [1.01-1.18] & 0.03 & $1.12[1.03-1.22]$ & 0.008 \\
\hline
\end{tabular}

Hierarchical analysis adjusted for center, Injury Severity Score, Extended IMPACT score, and mean ICP over the first $96 \mathrm{~h}$.

${ }^{a}$ Hazard ratios are calculated using mannitol as a categorical variable, and for 1 point increase in Injury Severity Score, Extended IMPACT Score, and $1 \mathrm{~mm} \mathrm{Hg}$ increase in mean ICP over the first $96 \mathrm{~h}$.

ICP, intracranial pressure; IMPACT, International Mission for Prognosis and Analysis of Clinical Trials in Traumatic Brain Injury; CI, confidence interval.

first day of a patient's stay, whereas HTS was administered on multiple days. Finally, we found that after adjustment for several key markers of illness severity and propensity to receive mannitol, the use of mannitol was associated with an approximate 2.5 -fold, but statistically non-significant $(p=0.06)$,,point estimate for an increased risk of death.

\section{Relationship to previous studies}

To our knowledge, no previous studies have described the epidemiology of osmotherapy use across multiple neurotrauma centers in different countries. In 2016, a systematic review of randomized controlled trials (RCTs) comparing HTS and mannitol concluded that HTS led to fewer failures to control ICP than mannitol, but that studies had been underpowered to detect a significant mortality difference. ${ }^{6}$ Moreover, the possible impact of osmotherapy choice on mortality has been assessed only as a secondary outcome in three of the six studies comparing these two types of osmotherapy. These three studies $^{9-11}$ reported mortality at variable time points ranging from in the hospital to 90 days or 6 months. However, illness severity scores were reported in only one study, ${ }^{11}$ and treatment with craniotomy, cooling, or barbiturate coma were only reported in another. ${ }^{12}$ The aggregate mortality from these 3 RCTs was 16 of 50 patients $(32 \%)$ treated with HTS, versus 21 of 55 patients $(38 \%)$ treated with mannitol. In summary, prospective studies so far looking at this question have been limited by small numbers, the fact that mortality was a secondary outcome, and by incomplete reporting of other key therapies.

\section{Implications of study findings}

Our study implies that mannitol and HTS are currently used as the sole osmotherapy agent in similar percentages in the early management of severe TBI across multiple centers in Europe, UK, and Australia. Daily patterns of osmotherapy administration differed markedly between the two osmotherapy groups, with man- nitol being administered largely on the first day of ICU stay, and HTS being used more consistently on each day, implying different clinician beliefs about the differing role and effects of the two osmotic agents. This differential prescribing does not appear to be explained by osmotherapy being given before emergency neurosurgery, with similar numbers of patients operated on in each group (23 patients in HTS group vs. 27 patients in the mannitol arm). Further, although only one concentration of mannitol was used $(20 \%)$ in almost all centers, large variation in HTS concentrations existed (from $3 \%$ to $23.5 \%$ ), implying the need to be specific when referring to HTS therapy and that there is no consensus regarding the optimal concentration to be used. Our results imply that mannitol may be given more frequently to patients with greater TBI severity, leading to a strong unadjusted association between its administration and mortality. Finally, the observation that, after adjustment for multiple markers of illness severity, mannitol remains a nearly significant predictive variable for mortality with a nearly 2.5-fold point estimate for an increase in the risk of death is striking. One possible explanation is that the choice of osmotherapy affects mortality. However, another possible explanation, given important differences between groups, is that this finding simply reflects differences in clinicians' beliefs about the role and effects of these two agents, incompletely accounted for by multi-variate analysis. This requires further investigation in controlled trials.

\section{Strengths and limitations}

Our study has several strengths. First, we included multiple centers from many countries, thereby increasing the external validity of our observations. Second, we provided detailed information on the dose, timing, and concentration of the osmotherapy used, thus enabling clinicians to relate such interventions to their practice. Third, we focused on the early phase of intervention with osmotherapy, where such intervention is most likely to have impact, thus maximizing our ability to see a difference if one exists.

Table 4. Unadjusted Hospital and ICU Mortality and Days Alive and Free From ICU or Hospital (Censored at Hospital Discharge)

\begin{tabular}{lccc}
\hline & HTS only $(\mathrm{n}=46)$ & Mannitol only $(\mathrm{n}=46)$ & $\mathrm{p}$ value \\
\hline Hospital mortality \% $(n)$ & $21.7(10)$ & $52.2(24)$ & 0.002 \\
ICU mortality \% $(n)$ & $15.2(7)$ & $47.8(22)$ & 0.001 \\
Days alive and free from ICU at 30 days & $13.4[5-18]$ & $0[0.0-13.3]$ & 0.004 \\
Days alive and free from hospital at 60 days & $25[0.0-37.3]$ & $0[0.0-5.5]$ & 0.16 \\
\hline
\end{tabular}

Data are presented as percentage $(n)$ or median [interquartile range].

ICU, intensive care unit; HTS, hypertonic saline. 


\section{Product-Limit Survival Estimates \\ With Number of Subjects at Risk}

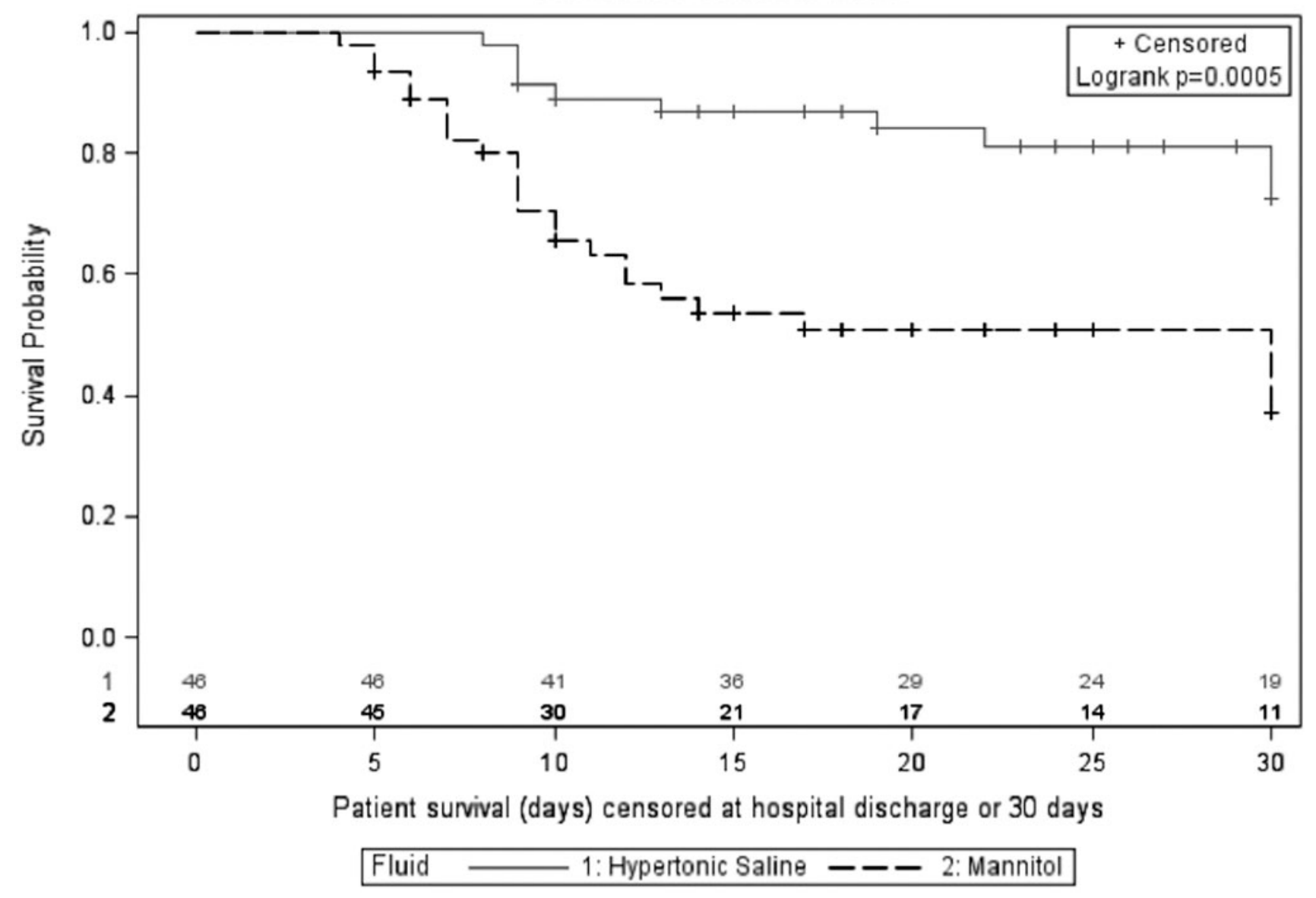

FIG. 3. Survival plot by osmotherapy agent, censored at hospital discharge or 30 days.

Fourth, we only compared patients where treatment was limited to one agent or the other, thereby removing the confounding effects of exposure to both agents. Moreover, we accounted for differences in baseline illness severity using several established and validated predictive scores (APACHE-II, IMPACT, and ISS) as well as other important elements of management, thus attenuating the effect of confounding on the relationship between osmotherapy and mortality.

Our study, however, has some limitations. It was not an RCT, and any association, unadjusted or adjusted, cannot be used to infer causality. Despite adjustment for multiple measures of illness severity, we may have failed to detect baseline differences between the patients, and it remains possible that mannitol use may have simply been a marker for greater clinician concern. Given that mannitol use was predominantly on the first day after injury (compared to the hypertonic saline group where the requirement for osmotherapy was in later days), this early ICH may also reflect an unmeasured difference between groups in patients' primary or secondary brain injury severity. Adjustment cannot account for unmeasured differences.

Of note, this is the first international, multi-center study to provide information on the association between osmotherapy choice and mortality, a key patient-centered outcome, which has previously not been formally assessed as primary outcome for osmotherapy in TBI patients. However, exact triggers for osmotherapy use were not recorded, meaning that we cannot differentiate between osmotherapy use as prophylaxis and treatment, nor determine whether clinicians may have used these two agents in different ways. Lack of follow-up of neurological outcome in survivors precludes any comments on whether the lower mortality in the HTS patients translated into meaningful long-term recovery. Moreover, lack of precise information about the causes of death precludes speculation about possible mechanisms of mannitol toxicity, if indeed any such toxicity exists. Of note, nephrotoxicity associated with mannitol exposure in neurosurgery and stroke patients ${ }^{13,14}$ has been described and an animal model of TBI has suggested that inflammation and apoptosis may be substantially lower with HTS than mannitol. ${ }^{15}$

Our study did not report on osmotherapy use in North American centers or developing countries, and we cannot comment on osmotherapy use in such settings. Finally, we do not have information about osmotherapy use before ICU admission; however, this has seldom been reported in studies of critical care osmotherapy use.

\section{Conclusion}

In conclusion, in an international, multi-center, observational study of early osmotherapy in patients with severe TBI, ICP monitoring, and $\mathrm{ICH}$, we found that the exclusive use of mannitol and HTS was similar, but also that patients treated with mannitol appeared to be more severely ill and that their unadjusted inhospital mortality was significantly greater. After adjustment for several markers of illness severity, mannitol use remained 
associated with a 2.5-fold point-estimate for an increase in risk of death. This point estimate was not significant. Our findings raise the possibility that choice of osmotherapy agents in severe TBI patients may affect survival, or, alternatively, simply reflect major differences in clinician beliefs about their different roles, and suggest the need for controlled investigation of osmotherapy agents in this population.

\section{Acknowledgment}

The authors acknowledge the considerable work of the following individuals who collected the data for this study: K. Long, Royal Melbourne Hospital, Parkville, Australia; A. Lozano, Erasme Hospital, Université Libre de Bruxelles, Brussels, Belgium; E. Saxby, The Alfred Hospital, Melbourne, Australia; A. Vargiolu, San Gerardo Hospital, Monza, Italy; A. Rodrigues, Le Kremlin Bicêtre, France; H. Quintard, Hôpital Pasteur, Nice, France; M. Del Rio, Hospital Clinic Universitari de Valencia, Valencia, Spain; A. Sisson, The Royal London Hospital, London, United Kingdom; G. Allen, Cambridge University Hospitals, Cambridge, United Kingdom; M. van der Jagt, Erasmus MC-University Medical Center, Rotterdam, The Netherlands; N. Baro, Campus Charité Mitte (CCM), Berlin, Germany; S. Wolf, Campus Charité Mitte (CCM), Berlin, Germany; M. Kofler, Medical University of Innsbruck, Innsbruck, Austria; D.W. Nelson, Karolinska Institute, Stockholm, Sweden.

\section{Author Disclosure Statement}

No competing financial interests exist.

\section{References}

1. Andrews, P.J., Sinclair, H.L., Rodriguez, A., Harris, B.A., Battison, C.G., Rhodes, J.K., and Murray, G.D.; Eurotherm3235 Trial Collaborators. (2015). Hypothermia for intracranial hypertension after traumatic brain injury. N. Engl. J. Med. 373, 2403-2412.

2. Cooper, D.J., Rosenfeld, J.V., Murray, L., Arabi, Y.M., Davies, A.R., D’Urso, P., Kossmann, T., Ponsford, J., Seppelt, I., Reilly, P., and Wolfe, R.; the DECRA Trial Investigators and the Australian and New Zealand Intensive Care Society Clinical Trials Group. (2011). Decompressive craniectomy in diffuse traumatic brain injury. N. Engl. J. Med. 364, 1493-1502.

3. Hutchinson, P.J., Kolias, A.G., Timofeev, I.S., Corteen, E.A., Czosnyka, M., Timothy, J., Anderson, I., Bulters, D.O., Belli, A., Eynon, C.A., Wadley, J., Mendelow, A.D., Mitchell, P.M., Wilson, M.H., Critchley, G., Sahuquillo, J., Unterberg, A., Servadei, F., Teasdale, G.M., Pickard, J.D., Menon, D.K., Murray, G.D., and Kirkpatrick, P.J.; RESCUEicp Trial Collaborators. (2016). Trial of decompressive craniectomy for traumatic intracranial hypertension. N. Engl. J. Med. 375, 1119-1130.

4. Carney, N., Totten, A.M., O'Reilly, C., Ullman, J.S., Hawryluk, G.W., Bell, M.J., Bratton, S.L., Chesnut, R., Harris, O.A., Kissoon, N., Rubiano, A.M., Shutter, L., Tasker, R.C., Vavilala, M.S., Wilberger, J., Wright, D.W., and Ghajar, J. (2017). Guidelines for the Management of Severe Traumatic Brain Injury, Fourth Edition. Neurosurgery $80,6-15$.
5. Oddo, M., Poole, D., Helbok, R., Meyfroidt, G., Stocchetti, N., Bouzat, P., Cecconi, M., Geeraerts, T., Martin-Loeches, I., Quintard, H., Taccone, F.S., Geocadin, R.G., Hemphill, C., Ichai, C., Menon, D., Payen, J.F., Perner, A., Smith, M., Suarez, J., Videtta, W., Zanier, E.R., and Citerio, G. (2018). Fluid therapy in neurointensive care patients: ESICM consensus and clinical practice recommendations. Intensive Care Med. 44, 449-463.

6. Burgess, S., Abu-Laban, R.B., Slavik, R.S., Vu, E.N., and Zed, P.J. (2016). A systematic review of randomized controlled trials comparing hypertonic sodium solutions and mannitol for traumatic brain injury: implications for emergency department management. Ann. Pharmacother. 50, 291-300.

7. Hays, A.N., Lazaridis, C., Neyens, R., Nicholas, J., Gay, S., and Chalela, J.A. (2011). Osmotherapy: use among neurointensivists. Neurocrit. Care 14, 222-228.

8. Wijayatilake, D.S., Talati, C., and Panchatsharam, S. (2015). The monitoring and management of severe traumatic brain injury in the United Kingdom: is there a consensus?: a national survey. J. Neurosurg. Anesthesiol. 27, 241-245.

9. Steyerberg, E.W., Mushkudiani, N., Perel, P., Butcher, I., Lu, J., McHugh, G.S., Murray, G.D., Marmarou, A., Roberts, I., Habbema, J.D., and Maas, A.I. (2008). Predicting outcome after traumatic brain injury: development and international validation of prognostic scores based on admission characteristics. PLoS Med. 5, e165; discussion, e165.

10. Cottenceau, V., Masson, F., Mahamid, E., Petit, L., Shik, V., Sztark, F., Zaaroor, M., and Soustiel, J.F. (2011). Comparison of effects of equiosmolar doses of mannitol and hypertonic saline on cerebral blood flow and metabolism in traumatic brain injury. J. Neurotrauma 28 , 2003-2012.

11. Vialet, R., Albanese, J., Thomachot, L., Antonini, F., Bourgouin, A., Alliez, B., and Martin, C. (2003). Isovolume hypertonic solutes (sodium chloride or mannitol) in the treatment of refractory posttraumatic intracranial hypertension: $2 \mathrm{~mL} / \mathrm{kg} 7.5 \%$ saline is more effective than $2 \mathrm{~mL} / \mathrm{kg} 20 \%$ mannitol. Crit. Care Med. 31, 1683-1687.

12. Jagannatha, A.T., Sriganesh, K., Devi, B.I., and Rao, G.S. (2016). An equiosmolar study on early intracranial physiology and long term outcome in severe traumatic brain injury comparing mannitol and hypertonic saline. J. Clin. Neurosci. 27, 68-73.

13. Deng, Y., Yuan, J., Chi, R., Ye, H., Zhou, D., Wang, S., Mai, C., Nie, Z., Wang, L., Zhai, Y., Gao, L., Zhang, D., Hu, L., Deng, Y., and Chen, C. (2017). The incidence, risk factors and outcomes of postoperative acute kidney injury in neurosurgical critically ill patients. Sci. Rep. 7, 4245.

14. Lin, S.Y., Tang, S.C., Tsai, L.K., Yeh, S.J., Shen, L.J., Wu, F.L., and Jeng, J.S. (2015). Incidence and risk factors for acute kidney injury following mannitol infusion in patients with acute stroke: a retrospective cohort study. Medicine (Baltimore) 94, e2032.

15. Soustiel, J.F., Vlodavsky, E., and Zaaroor, M. (2006). Relative effects of mannitol and hypertonic saline on calpain activity, apoptosis and polymorphonuclear infiltration in traumatic focal brain injury. Brain Res. 1101, 136-144.

Address correspondence to: James R. Anstey, MBBS, FRACP, FCICM Intensive Care Unit Royal Melbourne Hospital Parkville, 3050 Victoria Australia

E-mail: james.anstey@mh.org.au 